\section{Mental health}

Patients with mental health problems are frequent attenders in emergency settings and, regrettably, adequate provision for this group continues to fall well short of acceptable in most ED's, despite the acuity and vulnerability of these patients. Rightly or wrongly their definitive psychiatric care is often delayed if they have consumed alcohol or other substances, which can predispose them to even greater risk. A retrospective study by Urbanoski et al (see page 220) from Canada looked at frequent attenders with mental health disorders, delineating differences by number of attendances for substance use disorders (SUD) and predicted the receipt of follow-up services and 2-year mortality by level of ED use for SUD. Sadly but not surprisingly they found those with ED attendances for SUD were older and more likely to be men (ps <001) and as the number of attendances for SUD increased, the likelihood of receiving follow-up care (especially mental healthcare) declined, while the 2-year mortality steadily increased (ps <001) and this was even controlling for comorbidities and past service use. I am sure we will all identify with this Canadian experience; it's disheartening to read and a sad indictment of ours and the society's approach to patients with serious mental illness. This needs to change.

\section{The case for and against antibiotics}

We are all well aware that antimicrobial resistance, which is attributed in part to the overuse and misuse of these medications, is an increasingly serious threat to global public health. To minimise antibiotic resistance we all need to act judiciously in terms of need and benefit when prescribing. Most of us have deliberated as to whether antibiotics are necessary after incision and drainage of simple skin abscesses, indeed some schools of thought argue there is no need, so it was affirming to read a paper by Frazee (see page 277) from the USA describing the findings of two large multi-centre randomised controlled trials investigating the effect of off -patient antibiotics in uncomplicated skin abscesses. The studies by Talan et al and Daum et al unequivocally conclude that adjunctive antibiotics are beneficial for uncomplicated skin abscesses, although the magnitude of the benefit is small. Will this knowledge inform your prescribing? I suspect we will still consider each case on its own merit.

\section{Out of hospital cardiac arrest}

Prognostication in out-of-hospital cardiac arrests is known to be challenging. Very few studies have explored the decisions made by our ambulance colleagues in these situations, in terms of starting or stopping resuscitation. In the supportive environment of the ED we rarely consider the difficulties they encounter or how they manage these challenges. Thus it's an eye-opener to read in this issue a qualitative study by Anderson et al (see page 208) from New Zealand which explored decision making by ambulance personnel with respect to out-of-hospital cardiac arrests. They conducted semi-structured interviews with sixteen demographically diverse ambulance personnel employed in a variety of emergency response roles. Their findings suggest that confidence in a poor prognosis is only part of the decision-making process, but that better educational preparation and mentoring in managing the scene of a death to avoid prolonged or inappropriate resuscitation efforts would be beneficial. So, bear this in mind the next time you take handover of a cardiac arrest from a paramedic crew and you wonder why they continued resuscitation: they may not have felt able or confident to manage an out-of-hospital death.

\section{Effective sedation in resource limited hospitals}

Ketamine is commonly used for procedural sedation throughout the world but little is known about its safety in resource-constrained settings with minimal monitoring facilities. So ED clinicians in the developing world may be interested to read the findings of a prospective observational study of Ketamine procedural sedations conducted by Coralic et al (see page 214) in the main referral hospital in Dar es Salaam, Tanzania. They observed procedural sedation of 54 children and 45 adults; the most common indications for ketamine were burn management in children and orthopaedic procedures in adults. There were three deaths but these all occurred in severely injured patients, none of the deaths were thought to be caused by Ketamine. There were no serious adverse events and overall Ketamine was well tolerated even in high acuity non fasted patients. Patients and clinicians were both satisfied with the outcomes. If you are working in a resource limited environment (not the NHS!) have a read of this paper, it may offer the degree of reassurance you are looking for.

\section{Trauma}

Blunt thoracic aortic injury (BTAI) is an uncommon injury but it is the second leading cause of death behind traumatic brain injury (TBI) among blunt trauma patients. Death is usually due to haemorrhagic shock, timely diagnosis may enable early resuscitation but clinical features of impending shock and severity of injury are not always apparent. A systematic review by Bade-Boon and colleagues (see page 231) from Australia was undertaken to determine the proportion of patients with BTAI that present with abnormal vital signs. Nineteen studies were included with five selected for meta-analysis. Most cases of BTAI in this study were caused by road traffic accidents with mortality consistently higher among initially unstable patients. Interestingly, from this study they found vital signs to be poorly sensitive to ruling out aortic injury suggesting that ED clinicians need to maintain a high degree of clinical suspicion using imaging 'liberally' to prevent missed or delayed diagnosis. This paper is informative, so those of you working to improve trauma outcomes should have a read. 